\title{
Parallel Connected Multiple Drive System Using Small Auxiliary Inverter for Numbers of PMSM
}

\author{
Tsuyoshi Nagano and Jun-chi Itoh \\ Dept. of Energy and Environmental \\ Nagaoka University of Technology \\ Nagaoka, Niigata, Japan \\ ngn244@stn.nagaokaut.ac.jp \\ itoh@vos.nagaokaut.ac.jp
}

\begin{abstract}
This paper discusses a multi-parallel drive system for permanent magnet synchronous motors (PMSMs). This system proposes to use two different functions of inverter, a main inverter to drive parallel connected PMSMs and an auxiliary inverter to suppress the vibration of the motor speed. However, the power capacity of the auxiliary inverter depends on the speed response. In this paper, the performance of the proposed system is discussed. From the frequency characteristics, it is clarified that the power capacity of the auxiliary inverter is lower than $10 \%$ of the main inverter when the frequency components included in the speed command are less than 12 $\mathrm{rad} / \mathrm{s}$. As a result, the experimental results demonstrated that the proposed system can reduce the speed vibration from $400 \mathrm{r} / \mathrm{min}$ to nearly $0 \mathrm{r} / \mathrm{min}$ with damping control in the prototype of 1500-W PMSM drive system. The experimental results well agree with the theoretical analysis results.
\end{abstract}

Keywords - Permanent magnet synchronous motor, Parallel operation, Damping control.

\section{INTRODUCTION}

Recently, the adjustable speed drive techniques for the permanent magnet synchronous motor (PMSM) have attracted a lot of attentions among the researchers in terms of the energy saving [1]-[3]. PMSM has high efficiency and high power density in comparison with the induction motor (IM) because PMSM does not have the excitation circuit. On the other hand, IMs can be driven in parallel by using only one inverter. Parallel connected multiple induction motor drive systems are applied to industry in terms of cost reduction and simplify of the system, because this system only uses large power capacity inverter to multiple induction motors [4]-[7].

The efficiency of the motor drive system is improved by replacing IM with PMSM. However, it is difficult to drive the parallel connected PMSMs by using only one inverter because the torque vibration occurs due to the resonance between a synchronous reactance and the inertia moment [8]. When the parallel connected PMSMs are driven by one inverter, the current cannot be controlled corresponding to each pole of PMSM because the pole position is different from the pole position of other PMSMs. Therefore, the torque vibration will occur in this multiple motor drive system.
The damping control that uses the current feedback has been proposed [9]. This method suppresses the torque vibration with adding the vibrational component in the effective current to the output phase command of the voltage command. However, this method cannot be applied to the parallel connected PMSMs because one inverter cannot control each current corresponding to each pole of the PMSM.

There are some literatures which two PMSMs are driven by one power converters [10]-[15]. These converters can drive two PMSMs independently. However, these converters have some problems. For instance, the five-leg inverter [10]-[12] and the nineswitch inverter [13]-[14] is driven at low voltage utilization ratio in comparison with a case when one PMSM is driven by these converters because DC-link voltage is distributed to two PMSMs. Moreover, the current capacity of the switching device increases because there is a common leg. The four-leg inverter using the neutral point of the DC link capacitor is less number of the switches than the other topologies [15]. However, this converter requires the capacitor voltage balancing control because the capacitor neutral-point voltage is unbalanced by the motor current. Therefore, it is difficult to apply these converters to several motors because the output voltage of the inverter is limited and increasing the number of switching devices. Therefore, for this reason, these converters are not suitable for driving multi-parallel connected PMSMs. So, the authors have proposed a drive system for multi-parallel connected PMSMs by the auxiliary inverter and motor windings [16]-[17].

In this paper, the acceleration performance of the proposed system is evaluated from the frequency characteristic for the speed command, the acceleration test and the simulation results that two sets of parallel connected PMSMs are driven. From the frequency characteristics of the transfer function from the speed command to the rotational speed, it is clarified that the resonance is suppressed by the damping control applying to the auxiliary inverter. Moreover, the minimum power capacity of the auxiliary inverter is clarified from the frequency characteristics of the transfer function from the 
speed command to the output power of the auxiliary inverter.

This paper is organized as follows; first, the configuration and the control strategy of the proposed parallel drive system are introduced. Next, the suppression effect of the damping control from the frequency characteristics from the speed command to rotational speed in the proposed system. In addition, the stability of the proposed system with the damping control is clarified in the Routh-Hurwitz stability criterion. Next, the minimum power capacity of the auxiliary inverter clarified from the frequency characteristics from the speed command to the output power of each inverter in the proposed system. Finally, the simulation and experimental results demonstrate that the proposed system can drive multiple of parallel connected PMSMs effectively by suppressing the torque vibration.

\section{PROPOSED SYSTEM AND CONTROL STRATEGY}

Fig. 1 shows the configuration of the proposed system. In PMSMs, the auxiliary windings which are used in the damping control (with the auxiliary inverter) are placed in the slots together with the main windings. The proposed system uses two different power rating inverters The first one is the large power capacity inverter for the main windings to control the speed of the parallel connected PMSMs. The second one is a small power capacity inverter for the auxiliary windings to suppress the torque vibration. In term of the effectiveness of the proposed system, it is very important that the power capacity of the auxiliary inverter is enough small.

Fig. 2 shows the control block diagram of the proposed system. In the proposed system, the V/f control is applied to the main inverter and the field-oriented control and the damping control are applied to the auxiliary inverter. Each of the auxiliary inverter controls the current in auxiliary windings of the PMSM in order to suppress the torque and speed vibrations. Since these vibrations are caused by the phase difference between the rotational coordinates of the inverter and of the PMSM, it can be suppressed by the damping control in the auxiliary inverter.

Fig. 3 shows the relationship between the $\mathrm{d}-\mathrm{q}$ rotating frame and the $\gamma-\delta$ rotating frame for a $V / f$ control. In the control of the auxiliary invereter, the direction of the flux vector with permanent magnet is defined as d-axis as same as the conventional FOC. In the V/f control, the output voltage vector is defined as $\delta$-axis, the axis which lags by $\pi / 2 \operatorname{rad}$ from $\delta$-axis can be defined as $\gamma$-axis. The lag of the load angle $\varphi$ occurs between the d-q rotating frame and $\gamma-\delta$ rotating frame as shonwn in Fig. 3. Therefore, the load angle $\varphi$, rotational speed $\omega_{\text {re }}$ and the speed command $\omega^{*}$ can be expressed as

$$
p \varphi=\omega_{r e}-\omega^{*}
$$

where $p$ is differential operator.

When the vibration of the speed is caused by the resonance between the inertia moment and the synchronous reactance, the load angle $\varphi$ also is vibrating as shown (1). Then, the changes of the load angle $p \varphi$ is

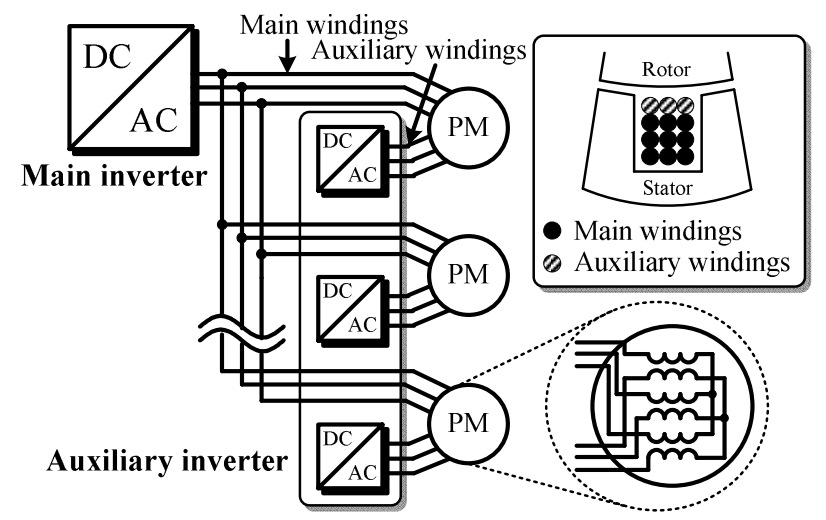

Fig. 1.Configuration of the proposed system. The proposed system uses two different power rating inverters, the large power capacity inverter to drive the motors and the small power capacity inverter to suppress the vibration.

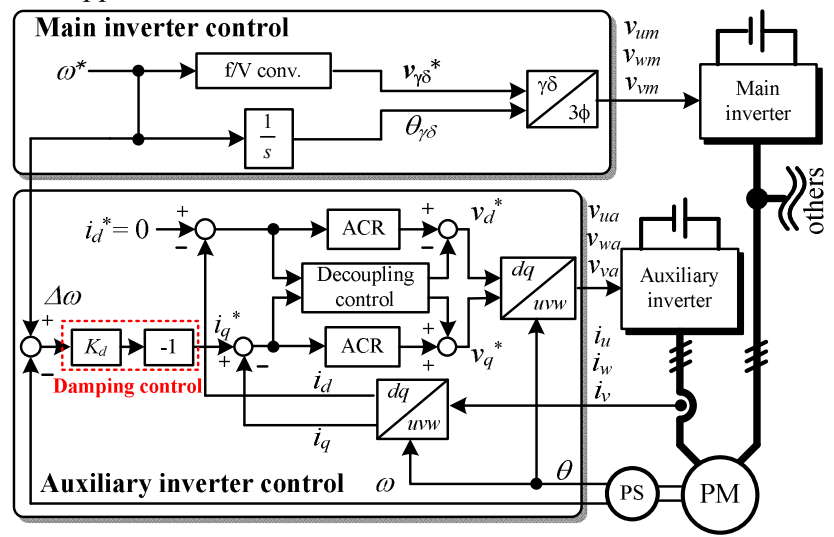

Fig. 2. Control block diagram of the proposed system. The $\mathrm{V} / \mathrm{f}$ control is applied to the main inverter. The auxiliary inverters uses the field-oriented control for the damping control.

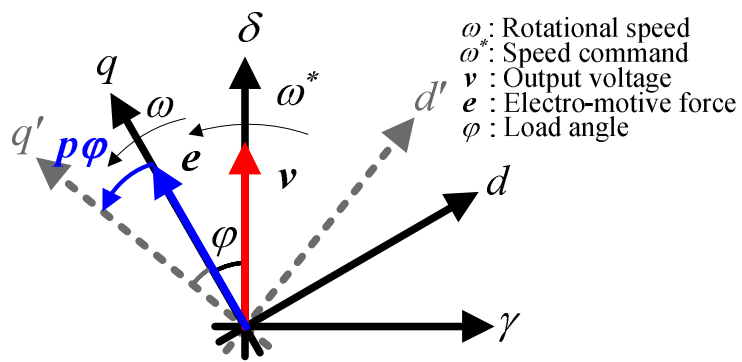

Fig. 3. Relationship between the d-q rotating frame and the $\gamma-\delta$ rotating frame. The $\gamma-\delta$ rotating frame lags by the load angle $\varphi$ from the d-q rotating frame.

the difference between the rotational speed and the speed command. In order to compensate the changes of the load angle $p \varphi$, the q-axis current command $i_{\mathrm{q}}{ }^{*}$ is calculated from the damping controller as shown in Fig. 2. As a result, the vibration of the speed and torque caused by the resonance are suppressed by compensating the changes of the load angle $p \varphi$ with the current controlled by FOC and the damping control.

\section{EVALUATION OF SUPPRESSION PERFORMANCE}

The damping control has to suppress the speed and torque vibration caused by the resonance between the inertia moment and the synchronous reactance. In this 
chapter, the suppression effect of the damping control is clarified from the frequency characteristics from the speed command to rotational speed in the proposed system.

Fig. 4 shows the simplified system in order to confirm the operation of the damping control. The proposed system uses the PMSM placed the auxiliary windings in the slots together with the main (conventional) windings, and so the mutual magnetic interference occurs between the main and the auxiliary windings. Due to the above reason, the control for the auxiliary inverter becomes complicated. Therefore, the proposed system is validated using a model where two PMSMs are connected in series via single shaft. Then, the rear end of the main PMSM is connected to the load machine. It means that the magnetic coupling was neglected in the simulation and the experiment.

The voltage equation of the motor that is connected to the main inverter is represented as the voltage equation in the $\gamma-\delta$ rotating frame because the main inverter is driven by the $\mathrm{V} / \mathrm{f}$ control. Note that the $\gamma-\delta$ rotating frame lags by the load angle $\varphi$ from the d-q rotating frame as shown in Fig. 3. On the other hand, the voltage equation of the motor that is connected to the auxiliary inverter is represented as the voltage equation in the $\mathrm{d}-\mathrm{q}$ reference frame because the auxiliary inverter control is FOC. Therefore, the voltage equation of the motor that is connected to the main inverter in the estimated rotating $\gamma$ $\delta$ frame is given by

$\left[\begin{array}{c}v_{M \gamma} \\ v_{M \delta}\end{array}\right]=\left[\begin{array}{cc}R_{M}+p L_{d M} & -\omega^{*} L_{q M} \\ \omega^{*} L_{d M} & R_{M}+p L_{q M}\end{array}\right]\left[\begin{array}{l}i_{M \gamma} \\ i_{M \delta}\end{array}\right]+\omega_{r e} \psi_{m M}\left[\begin{array}{l}\sin \varphi \\ \cos \varphi\end{array}\right]$

On the other hand, the voltage equation of the motor that is connected to the auxiliary inverter in $d-q$ reference frame can be given by

$\left[\begin{array}{c}v_{A d} \\ v_{A q}\end{array}\right]=\left[\begin{array}{cc}R_{A}+p L_{d A} & -\omega_{r e} L_{q A} \\ \omega_{r e} L_{d A} & R_{A}+p L_{q A}\end{array}\right]\left[\begin{array}{c}i_{A d} \\ i_{A q}\end{array}\right]+\left[\begin{array}{c}0 \\ \omega_{r e} \psi_{m A}\end{array}\right]$

Moreover, torque and speed equations can be given by

$$
\begin{aligned}
& T=T_{M}-T_{A}=\frac{3}{2} P_{f M} \psi_{m M}\left(i_{M \gamma} \sin \varphi+i_{M \delta} \cos \varphi\right)-\frac{3}{2} P_{f A} \psi_{m A} i_{A q} \\
& p \omega_{r e}=\frac{1}{J}\left(P_{f M} T_{M}-P_{f A} T_{A}\right)
\end{aligned}
$$

where $p$ is differential operator, $R$ is armature resistance, $L$ is synchronous reactance, $P_{\mathrm{f}}$ is pairs of the pole, $\Psi_{\mathrm{m}}$ is magnet flux linkage, $\varphi$ is the load angle, and $J$ is the inertia moment of motors, Suffix ' $A$ ' represents the parameter of the motor that is connected to the auxiliary inverter, ' $M$ ' represents the parameter of the motor that is connected to the main inverter.

Equations (2) and (3), (4) are non-linear so that these
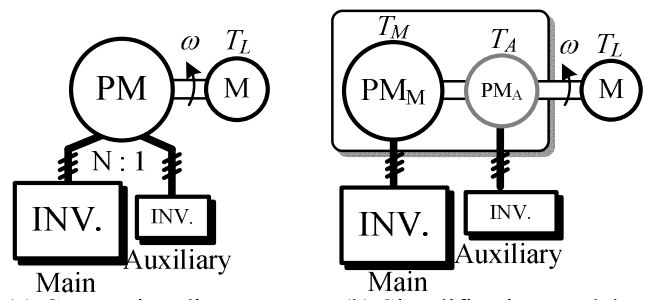

(a) Connection diagram

(b) Simplification model

Figs.4 Analysis and verification model of the PMSM in addition the auxiliary windings for damping control.

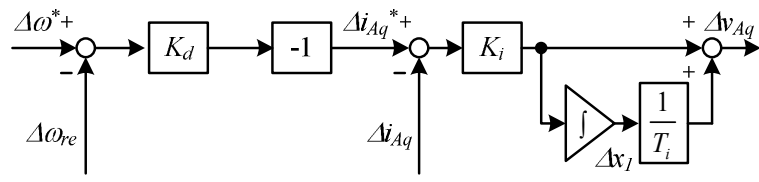

Fig. 5. State variable diagrams of the auxiliary inverter control. If the current control response is faster than the damping control response, the loop gain in the current control is 1 . Therefore, the q-axis voltage command $\Delta v_{A q}{ }^{*}$ can be given by (10).

equations are linearized about the steady state. The state equation after the linearization can be given by (6).

Equation (6) shows the $6^{\text {th }}$ order of the state equation that is complicated to evaluate the stability. In order to simplify this equation, it is assumed that the mechanical time constant is larger than electrical time constant, then (6) can be approximated as the $2^{\text {nd }}$ order state equation, as (7).

$$
\begin{aligned}
& {\left[\begin{array}{c}
p \Delta \omega_{r e} \\
p \Delta \varphi
\end{array}\right]=\left[\begin{array}{ccc}
-\frac{3}{2} \frac{P_{f M}{ }^{2} \psi_{m M}}{J} \frac{\psi_{m M} R_{M}}{\omega_{0}^{2} L_{d M} L_{q M}} & \frac{3}{2} \frac{P_{f M}{ }^{2} \psi_{m M}{ }^{2}}{J L_{q M}} \\
-1
\end{array}\right]\left[\begin{array}{c}
\Delta \omega_{r e} \\
\Delta \varphi
\end{array}\right]} \\
& +\left[\begin{array}{ccc}
\frac{3}{2} \frac{P_{f}^{2} \psi_{m M} R_{M}}{J \omega_{0}^{2} L_{d} L_{q}} & -\frac{3}{2} \frac{P_{f}^{2} \psi_{m A}}{J R_{A}} & 0 \\
0 & 0 & 1
\end{array}\right]\left[\begin{array}{c}
\Delta v_{M \delta} \\
\Delta v_{A q} \\
\Delta \omega_{1}
\end{array}\right]
\end{aligned}
$$

where suffix ' 0 ' is the steady state value.

Fig. 5 shows the state variable diagrams of the auxiliary inverter control that is applying the damping control. In Fig. 5, the q-axis voltage command $\Delta v_{\mathrm{Aq}}{ }^{*}$, it is assummed that the loop gain in the current control is 1 (assuming that the current control response is faster than the damping control response), can be given by

$\Delta v_{A q}=-K_{d}\left(\Delta \omega^{*}-\Delta \omega_{r e}\right)$

$p \Delta x_{1}=0$

where $K_{\mathrm{d}}$ is the damping gain.

Moreover, in Fig. 3 , the $\delta$-axis voltage command $\Delta v_{\mathrm{M} \delta} *$ and the $\gamma$-axis voltage command $\Delta v_{\mathrm{M} \gamma}{ }^{*}$ of the main

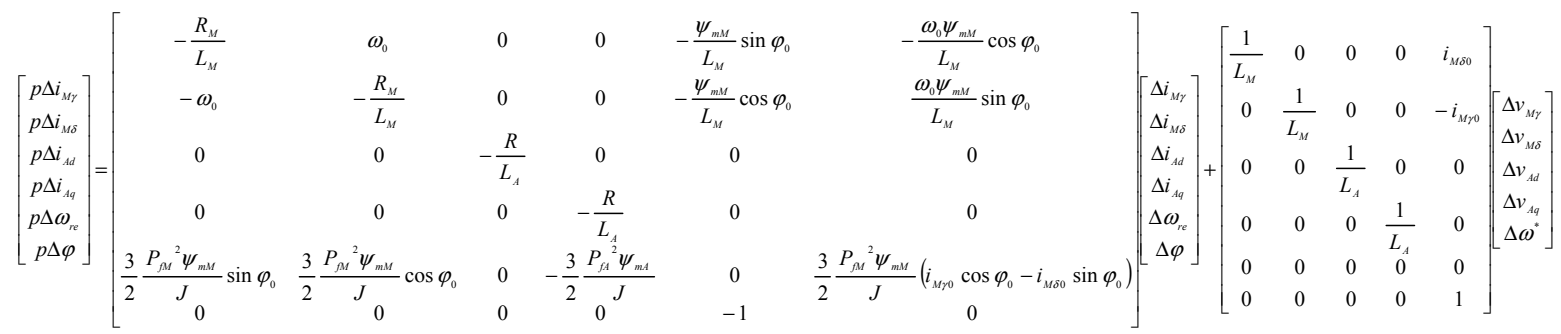


inverter are given by

$\Delta v_{M \gamma}=0$

$\Delta v_{M \delta}=\psi_{m M} \Delta \omega^{*}$

Assuming as $\delta_{0}=0, i_{M \gamma 0}=0, i_{M \delta 0}=0, \omega L$ is larger than $R$, substituting (8), (10) and (11) into (7) yields the state equation of

$$
p \mathbf{x}=\mathbf{A x}+\mathbf{B u}
$$

where $\mathbf{x}=\left[\begin{array}{ll}\Delta \omega_{\text {re }} & \Delta \delta\end{array}\right], \mathbf{u}=\Delta \omega^{*}$

$$
\mathbf{A}=\left[\begin{array}{cc}
-\frac{3}{2} \frac{P_{f M}{ }^{2} \psi_{m M}}{J} K_{d} & \frac{3}{2} \frac{P_{f M}{ }^{2} \psi_{m M}{ }^{2}}{J L_{q M}} \\
-1 & 0
\end{array}\right], \mathbf{B}=\left[\begin{array}{c}
\frac{3}{2} \frac{P_{f M}{ }^{2} \psi_{m M}}{J} K_{d} \\
1
\end{array}\right]
$$

Fig. 6 shows the frequency characteristic of the transfer function from the speed command to rotational speed of the V/f control from the main motor. Table 1 shows the analysis and experimental conditions. In Fig. 6 , the magnitude becomes lower at the resonance frequency $\omega_{\mathrm{n}}$ by applying the damping control in comparison to the proposed system without the damping control. It means that the suppression effect becomes higher.

The transfer function is derived from the $2^{\text {nd }}$ state equation of (12). The speed command $\Delta \omega^{*}$ to the rotational speed $\Delta \omega_{\mathrm{re}}$ transfer function is given by

$$
\begin{aligned}
\frac{\Delta \omega_{r e}}{\Delta \omega^{*}}= & \frac{\frac{3}{2} \frac{P_{f}^{2} \psi_{m M}}{J}}{s^{2}+\frac{3}{2} \frac{P_{f}^{2} \psi_{m M}}{J} K_{d} s+\frac{3}{2} \frac{P_{f}^{2} \psi_{m M}^{2}}{J L_{q M}}} \times \\
& \left\{\frac{\psi_{m M}}{L_{q M}}-s\left(K_{d}+\frac{\left(i_{M \gamma 0} \cos \varphi_{0}+i_{M \delta 0} \sin \varphi_{0}\right)}{\omega_{0}}-\frac{\psi_{m M}}{L_{q M} \omega_{0}} \sin \varphi_{0}\right)\right\}
\end{aligned}
$$

The damping factor $\zeta$ and the natural angular frequency $\omega_{\mathrm{h}}$ are given by

$$
\begin{aligned}
& \zeta=\sqrt{\frac{3}{2}} \frac{P_{f M} K_{d}}{2} \sqrt{\frac{L_{q M}}{J}} \\
& \omega_{n}=\sqrt{\frac{3}{2}} \frac{P_{f M} \psi_{m M}}{\sqrt{J L_{q M}}} \cdots \cdots
\end{aligned}
$$

Without the damping control $(\zeta=0)$, the magnitude at the resonance frequency rises. By contrast, by applying

\begin{tabular}{|c|c|c|}
\hline & $\mathrm{PM}_{\mathrm{M}}$ & $\mathrm{PM}_{\mathrm{A}}$ \\
\hline Rated power [W] & 1500 & 750 \\
\hline Rated speed $\left[\mathrm{min}^{-1}\right]$ & \multicolumn{2}{|c|}{1800} \\
\hline Rated current [A] & 8.2 & 4 \\
\hline Number of pole pairs & 3 & 3 \\
\hline Armature resistance $[\Omega]$ & 1.55 & 1.98 \\
\hline d-axis inductance $[\mathrm{mH}]$ & 11.5 & 15.2 \\
\hline q-axis inductance $[\mathrm{mH}]$ & 23 & 33.2 \\
\hline Electro-motive force constant [Vs/rad] & 0.368 & 0.338 \\
\hline Inertia moment $\left[\mathrm{kgm}^{2}\right]$ & 0.0051 & 0.0026 \\
\hline Rotational speed in stationary state $[\mathrm{rad} / \mathrm{s}]$ & \multicolumn{2}{|c|}{900} \\
\hline
\end{tabular}
the damping control $(\zeta=0.3)$, the magnitude at the resonance frequency decreases to $5 \mathrm{~dB}$. The high suppression effect is obtained by increasing the damping gain as shown (14) because the damping factor increases.
Table 1. Analysis and experimental conditions

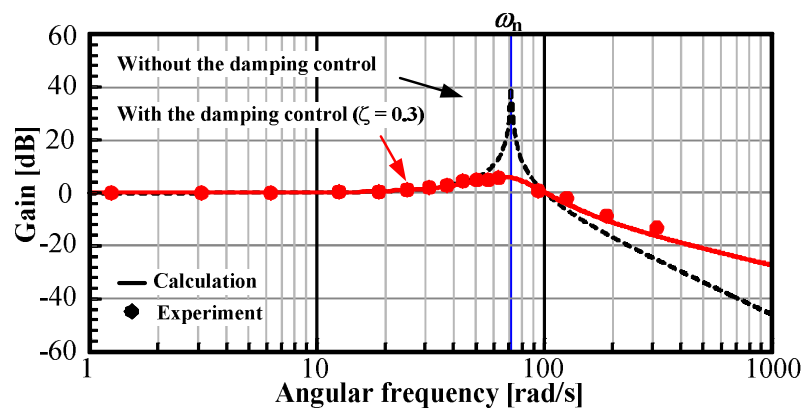

Fig. 6. Frequency characteristics of the speed command to the rotational speed in the proposed system. Table I shows the evaluated condition. By applying the damping control, the magnitude at the resonance frequency decreases to $5 \mathrm{~dB}$.

Therefore, the suppression effect of the damping control is clarified from the frequency characteristics from the speed command to rotational speed in the proposed system. The proposed damping control can suppress the resonance between the moment inertia and synchronous reactance as show in Fig. 6. In the experiment, when the constant speed command adds sinusoidal wave as the speed command, the amplitude in the frequency of the sinusoidal wave is drawn by observing the speed response. The gain between the speed commands and rotational speed in the experimental results has been plotted in Fig. 6 as dot marks. The experimental results well agree with the analysis results.

\section{STABILITY OF PROPOSED SYSTEM}

In the previous section, it is confirmed that the PMSM can suppress the resonance by applying the damping control in the proposed system. In this chapter, the stability of the proposed system with the damping control

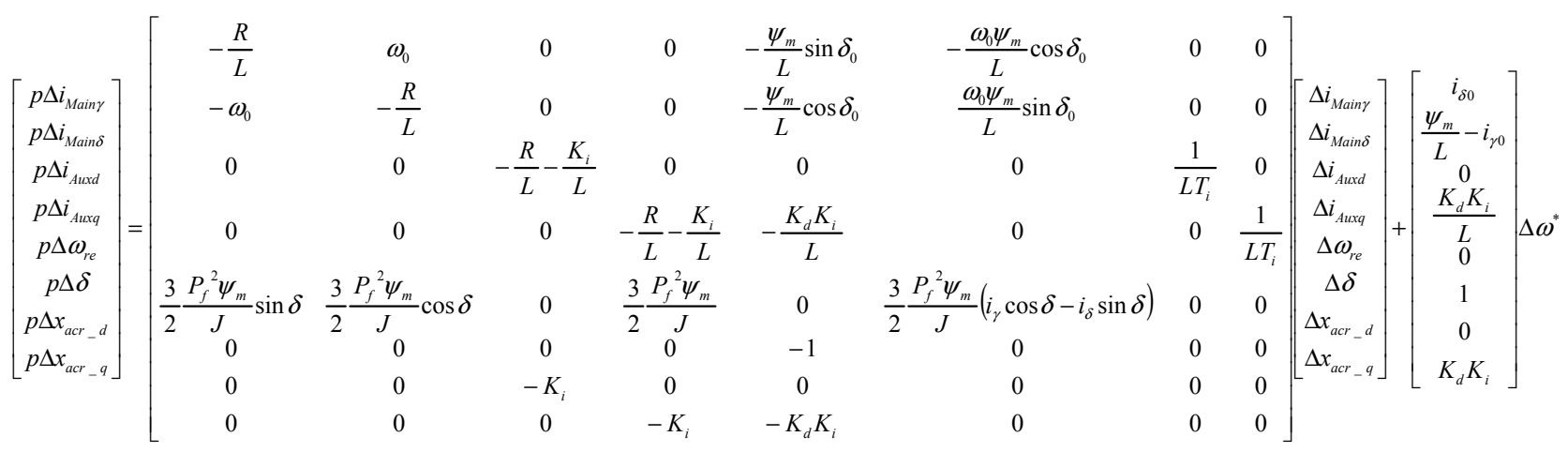


is clarified in the Routh-Hurwitz stability criterion.

The state equation of the proposed system which is applied with the damping control is given by (16).

There are two integrators in the auxiliary inverter control because the field-oriented control has d-axis and q-axis current control as shown in Fig. 3. Thus, the state variables becomes from six to eight state variables when the auxiliary inverter is applied with the damping control.

The Routh-Hurwitz stability criterion is based on ordering the coefficients of the characteristic polynomial which have the real coefficients of (17).

$$
a_{0} s^{n}+a_{1} s^{n-1}+\cdots+a_{n-1} z+a_{n}=0
$$

The proposed system is stable when all roots the characteristic polynomial of (17) is negative. In Hurwitz stability criterion, the necessary and sufficient conditions for all roots of the characteristic polynomial of (17) are as follows;

1) There are All coefficients $\left(a_{0}, a_{1}, a_{2}, \ldots, a_{\mathrm{n}}\right)$.

2) All coefficients have same sign.

3) All Hurwitz determinants $\left(\Delta_{1}, \Delta_{2}, . ., \Delta_{\mathrm{n}-1}\right)$ are positive.

$$
\begin{aligned}
\Delta_{1} & =a_{1} \\
\Delta_{2} & =\left|\begin{array}{ll}
a_{1} & a_{3} \\
a_{0} & a_{2}
\end{array}\right| \\
\Delta_{n-1} & =\left|\begin{array}{ccccc}
a_{1} & a_{3} & a_{5} & \cdots & a_{2 n-3} \\
a_{0} & a_{2} & a_{4} & \cdots & a_{2 n-4} \\
0 & a_{1} & a_{3} & \cdots & a_{2 n-5} \\
0 & a_{0} & a_{2} & \cdots & a_{2 n-6} \\
0 & 0 & a_{1} & \cdots & a_{2 n-7} \\
\vdots & \vdots & \vdots & \ddots & \vdots \\
0 & 0 & 0 & \cdots & a_{n-1}
\end{array}\right|
\end{aligned}
$$

Table 2 shows calculation of Hurwitz determinants and Table 3 shows the coefficient of the characteristic polynomial in the state equation of the proposed system which is applied with the damping control of (16). The analysis condition is shown in Table 1. All Hurwitz determinants are positive as shown Table 2. Moreover, there are All coefficient which have same sign. Therefore, because of the necessary and sufficient conditions, the proposed system is stable.

\section{MINIMUM POWER CAPACITY OF AUXILIARY INVERTER}

In the previous section, it is confirmed that the PMSM can be stabilized by applying the damping control in the proposed system. However, if the damping gain $K_{d}$ increases to obtain the larger suppression effect, the output power of the auxiliary inverter becomes larger. Moreover, the power capacity of the auxiliary inverter depends on the speed command response. Thus, in this section, the minimum power capacity of the auxiliary inverter is clarified from frequency characteristics.

Fig. 7 shows the frequency characteristic of the transfer function from speed command to output power of the main inverter, the auxiliary inverter, and the sum of the inverters in the proposed system. In the proposed system, the main torque cannot be controlled directly due
Table 2 Calculation of Hurwitz determinants

\begin{tabular}{c|cc}
\hline Hurwitz determinants & \multicolumn{2}{|c}{ Results } \\
\hline \hline$\Delta_{1}$ & 8458 & $>0$ \\
$\Delta_{2}$ & $2.138 \times 10^{11}$ & $>0$ \\
$\Delta_{3}$ & $9.616 \times 10^{21}$ & $>0$ \\
$\Delta_{4}$ & $5.479 \times 10^{35}$ & $>0$ \\
$\Delta_{5}$ & $9.657 \times 10^{51}$ & $>0$ \\
$\Delta_{6}$ & $5.691 \times 10^{70}$ & $>0$ \\
$\Delta_{7}$ & $3.486 \times 10^{91}$ & $>0$
\end{tabular}

Table 3 Coefficient of the characteristic polynomial

\begin{tabular}{c|cc|c}
\hline Coefficients & & Coefficients & \\
\hline \hline$a_{0}$ & 1 & $a_{4}$ & $7.404 \times 10^{13}$ \\
$a_{1}$ & 8458 & $a_{5}$ & $2.765 \times 10^{16}$ \\
$a_{2}$ & $3.339 \times 10^{7}$ & $a_{6}$ & $9.413 \times 10^{18}$ \\
$a_{3}$ & $6.866 \times 10^{10}$ & $a_{7}$ & $9.888 \times 10^{20}$ \\
& & $a_{8}$ & $9.143 \times 10^{22}$
\end{tabular}

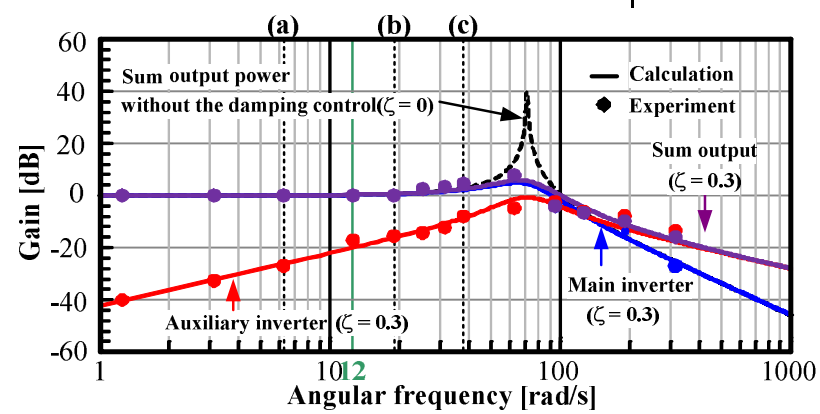

Fig. 7. Frequency characteristics of the transfer function from speed command to output power of the main inverter, the auxiliary inverter, and the sum of the inverters in the proposed system.

to the $\mathrm{V} / \mathrm{f}$ control. However, by calculating the torque transfer function from the speed one, the frequency characteristic of each output power is derived. If the electrical and mechanical losses are neglected, the output power of the auxiliary inverter depends on the mechanical output of the motor. In other words, the transfer function of the output power for the auxiliary inverter is derived from the mechanical output transfer function of the motor. However, the mechanical output is nonlinear because the mechanical output is the product of two variables, the rotational speed and the torque. Due to this reason, the output power transfer function of the auxiliary inverter is derived after the mechanical output is linearized. The speed command to the output power of the auxiliary inverter transfer function is given by

$$
\frac{\Delta P_{A}}{\Delta \omega^{*}}=\frac{s^{2} \omega_{0} K_{d} \psi_{m A}}{s^{2}+\frac{P_{f M}{ }^{2} \psi_{m M}}{J} K_{d} s+\frac{P_{f M}{ }^{2} \psi_{m M}{ }^{2}}{J L_{q M}}}
$$

In Fig. 7, the output power of the auxiliary inverter rises near the resonance frequency. On the other hand, when the frequency components into the speed command are lower and lower, the output power of the auxiliary inverter is suppressed more. In other words, the auxiliary inverter does not contribute steady state i.e. constant speed. Nevertheless, although the output power of the 
main inverter is constant because the main inverter drives the motor. Note that the damping factor is designed by the equations which are derived by the authors.

Figs. 8 show the experimental results that each output power is measured at each operating point in Fig. 5 when 0.5 p.u. of the constant speed command adds sinusoidal wave as the speed command. Assuming that the inverter output voltage is equal to the voltage command, each output power is calculated from the voltage command and the measured current. The waveforms in Fig. 8(a), (b) and (c) mention the operation at the point (a), (b) and (c) in Fig. 7, respectively. Comparing with each result, the amplitude ratio between the output power of the auxiliary inverter and the main inverter is changed corresponding to the frequency components included in the speed command. When the frequency component included in the speed command is lower than $32 \mathrm{rad} / \mathrm{s}$, the output power of the auxiliary inverter can become lower than $10 \%$ of the main inverter as shown Fig. 7. This boundary condition is decided by inertia moment $J$, q-axis inductance $L_{q M}$, magnet flux linkage $\Psi_{\mathrm{m}}$ and the damping gain $K_{d}$ as shown (14). When the damping gain $K_{d}$ increases, the output power of the auxiliary inverter increase from $10 \%$ of the main inverter at $32 \mathrm{rad} / \mathrm{s}$ because the damping factor increases. Therefore, the results show that the power capacity of the auxiliary inverter can be designed to nearly several percent of the main inverter when the proposed system is applied to the application which requires a slow speed response such as fan applications. In this session, in order to confirm the validity of proposed system in terms of acceleration which means the rate of the speed command, the relationship between the output power of the auxiliary inverter and the overshoot of the rotational speed for speed command is demonstrated in the experiment.

The output power of the auxiliary inverter was discussed by frequency characteristics. However, the acceleration or deceleration time is set as the speed command in practically. In this session, the output power of the auxiliary inverter is discussed by acceleration which means the rate of the speed command.

The time response of the rotational speed is derived from the inverse Laplace transform of the ramp response of (13). In this section, in order to simplify (13), it is assumed as $\varphi_{0}=0, i_{M \gamma 0}=0$ and $i_{M \delta 0}=0$ in (13). Moreover, the overshoot of the rotational speed can be derived from this time response, as given by

$$
\begin{gathered}
\Delta \omega_{\text {re_overshoot }}=\frac{\alpha \sin \left(\omega_{n} t_{\text {peak }} \sqrt{1-\zeta^{2}}\right)}{\omega_{n} \sqrt{1-\zeta^{2}} \exp \left(\zeta \omega_{n} t_{\text {peak }}\right)} \\
t_{\text {peak }}=\frac{1}{2} \frac{\log \left(2 \zeta^{2}-1+2 \zeta \sqrt{\zeta^{2}-1}\right)}{\omega_{n} \sqrt{\zeta^{2}-1}} \ldots
\end{gathered}
$$

where $\alpha\left[\mathrm{rad} / \mathrm{s}^{2}\right]$ is acceleration.

The overshoot of the rotational speed can be calculated by using (19).

Similarly, the time response of the output power of the auxiliary inverter during the acceleration is also derived from the inverse Laplace transform of the ramp response

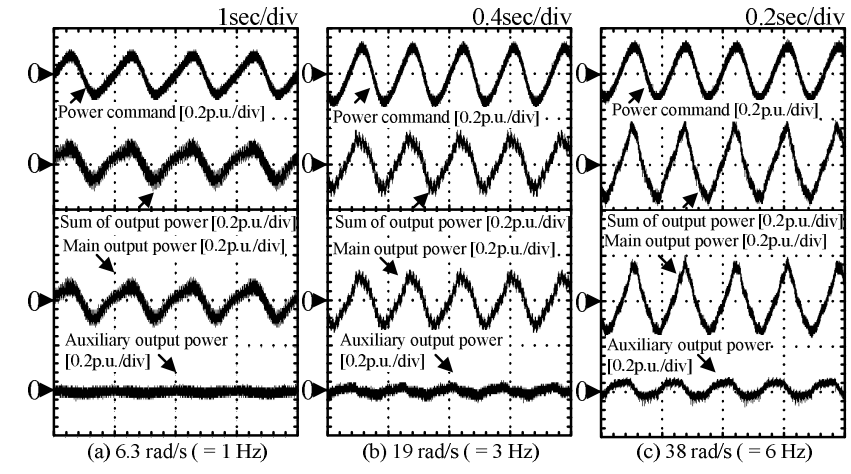

Figs. 8. Experimental results that each output power is measured at each operating point ((a) is at $1 \mathrm{~Hz},(\mathrm{~b})$ is at 3 $\mathrm{Hz},(\mathrm{c})$ is $6 \mathrm{~Hz}$ ) in Fig. 7 when 0.5 p.u. of the constant speed command adds sinusoidal wave as the speed command.

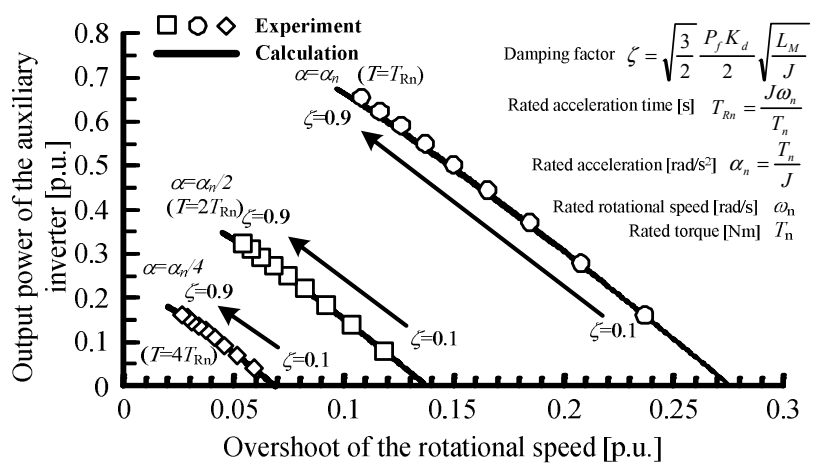

Fig. 9. Relationship between the output power of the auxiliary inverter and the overshoot of the rotational speed the against speed command. Table 1 shows also the evaluated condition. The higher suppression effect is obtained in exchange for the increase of the output power of the auxiliary inverter by increasing the damping gain.

of (18). In this section, in order to simplify (18), it is assumed as $\delta_{0}=0, i_{M \gamma 0}=0, i_{M \delta 0}=0$ in (18). Moreover, the maximum output power of the auxiliary inverter can be derived from this time response, as given by

$$
\Delta P_{\text {aPeak }}=\frac{P_{f} \psi_{m A} K_{d} \omega_{0} \alpha \sin \left(\omega_{n} \sqrt{1-\zeta^{2}} t_{\text {peak }}\right)}{\omega_{n} \sqrt{1-\zeta^{2}} \exp \left(\zeta \omega_{n} t_{\text {peak }}\right)}
$$

The maximum output power of the auxiliary inverter can be calculated by (21).

Fig. 9 shows the relationship among the damping gain $K_{d}$, the maximum output power of the auxiliary inverter during the acceleration and the overshoot of the rotational speed when the PMSM is accelerated during one, half, quarter of the rated acceleration $\alpha_{\mathrm{n}}$ on the condition as shown in Table 1. The damping gain is standardized by the damping factor $\zeta$ as given by (14). The output power of the auxiliary inverter rises with the increase of the damping factor. On the other hand, the overshoot of the rotational speed decreases with the increase of the damping factor, as shown in Fig. 9. The maximum output power of the auxiliary inverter (i.e. the power capacity of the auxiliary inverter) depends on the acceleration factor $\alpha$ in the speed command as shown in Fig. 9. The experimental results well agree with the calculation. 


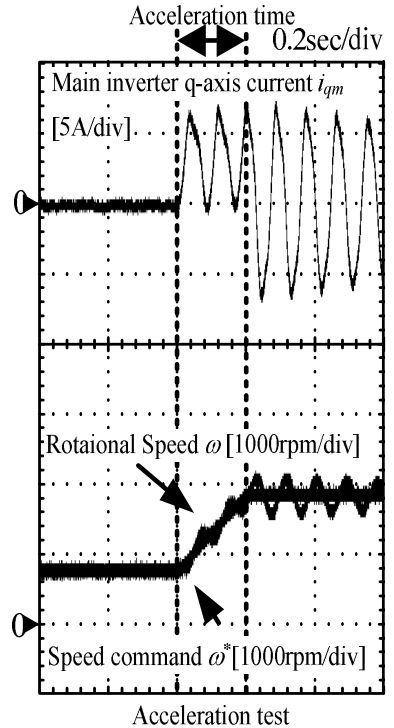

(a) without damping control

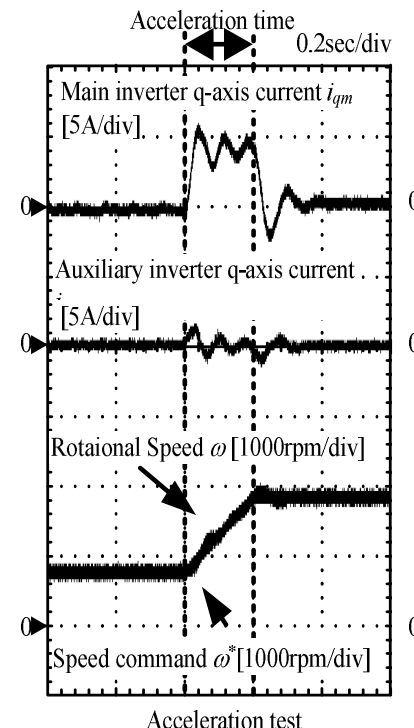

(b) with damping control.

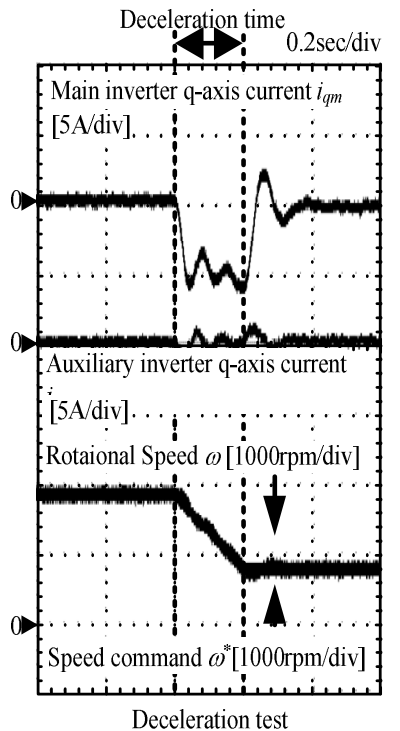
Fig. 10. Acceleration and deceleration test without/with damping control in motor-generator set. (a) After the acceleration,
the $400 \mathrm{r} / \mathrm{min}$ - speed vibration is maintained. (b) The speed vibration is reduced from $400 \mathrm{r} / \mathrm{min}$ to nearly $0 \mathrm{r} / \mathrm{min}$ in compared with (a)

\section{MOTOR DRIVE PERFORMANCE EVALUATION}

In this chapter, in order to confirm the effectiveness of the damping control, the experiments are conducted with a motor-generator set.

Fig. 10 shows the experimental results that illustrate the motor speed vibration when the proposed system is applied (a) without the damping control and (b) with the damping control $(\zeta=0.3)$ in an acceleration and deceleration test. In this experiment, it is difficult to measure the torque response directly. Therefore, the speed vibration is evaluated instead of the torque response. In Fig. 10(a), the proposed system is implemented without the damping control. The speed vibration occurs during the acceleration. After the acceleration, a $400 \mathrm{r} / \mathrm{min}$ of speed vibration and the 10 $A_{p-p}$ of current vibration in q-axis of the main inverter are maintained. On the other hand, Fig. 10(b) demonstrates the experimental results, where the proposed system is implemented with the damping control $(\zeta=0.3)$. The effectiveness of the auxiliary inverter from the results confirms that the speed vibration is reduced from 400 $\mathrm{r} / \mathrm{min}$ to nearly $0 \mathrm{r} / \mathrm{min}$ in compared with the acceleration test of Fig. 10(a). The $10 \mathrm{~A}_{\mathrm{p}-\mathrm{p}}$ of the current vibration in the q-axis of the main inverter is suppressed as well. Then, the output power of the auxiliary inverter is suppressed to $13 \%$ of the main inverter when the damping factor is designed at 0.3 . In the same way as the acceleration test of Fig. 10(a), after the deceleration, a $500 \mathrm{r} / \mathrm{min}$ of speed vibration and the $15 \mathrm{~A}_{\mathrm{p}-\mathrm{p}}$ of current vibration in q-axis of the main inverter are maintained as shown in the deceleration test of Fig. 10(a). In contrast, it is confirmed that, as shown the deceleration test of Fig. 10 (b), the speed vibration is reduced from $500 \mathrm{r} / \mathrm{min}$ to nearly $0 \mathrm{r} / \mathrm{min}$ and the current vibration is reduced from $15 \mathrm{~A}_{\mathrm{p}-\mathrm{p}}$ to $0 \mathrm{~A}_{\mathrm{p}-\mathrm{p}}$ in compared to Fig. 10(a) in the same way as acceleration test. Nevertheless, it is confirmed that the q-axis current of the auxiliary inverter flows only during acceleration and deceleration. Moreover, the maximum q-axis current of the auxiliary inverter is $20 \%$ of the q-axis current of the main inverter. Therefore, it is confirmed that the auxiliary inverter can suppress the speed vibration via auxiliary windings with a small q-axis current of the auxiliary inverter even if in the acceleration and deceleration test.

\section{EVALUATION OF PARALLEL DRIVE SYSTEM}

In previous chapter, the effectiveness of the damping control in the proposed system is discussed. This chapter discusses the effectiveness of the proposed system when two PMSM are driven.

Fig. 9 shows the simulation models that are used to verify the operation of two sets of parallel connected PMSM. Since it is difficult to construct the prototype of the drive system using two PMSMs, the simulation is used to evaluate the validity of the proposed controller as first step.

Fig. 10 shows the simulation results when two sets of parallel connected PMSMs are driven by the proposed system with the damping control as shown in Fig. 8. Smooth acceleration progresses are confirmed in the two set of parallel connected PMSMs. Besides, when the rated motor speed, load step applies to PMSM1 at $0.38 \mathrm{~s}$, and later also applies to PMSM2 at $0.42 \mathrm{~s}$, the operation of the two auxiliary inverters can be observed from the output power. The maximum output power of the auxiliary inverter is approximately 0.25 p.u. of the rated power of the main inverter. Although the power capacity of main inverter increases with the increase of parallel units, the power capacity of auxiliary inverter does not change according to the numbers of parallel units. 


\section{CONCLUSION}

This paper discusses the acceleration performance of the proposed parallel connected multiple PMSM drive system from the frequency characteristics and the acceleration test. From the frequency characteristics of the speed response, it was clarified that the resonance is suppressed by applying the damping control. In addition, the output power of the auxiliary inverter is suppressed to $10 \%$ of the main motor in the speed response of lower than $32 \mathrm{rad} / \mathrm{s}$ in the prototype of 1500-W PMSM drive system. The results show that the power capacity of the auxiliary inverter is designed to nearly several percent of the main inverter when the proposed system is applied to the application which requires a slow speed response such as fan applications.

In the future work, the driving several units of the motor-generator in parallel with the proposed system drives will be evaluated in the experimental system.

\section{ACKNOWLEDGMENT}

A part of this study was supported by Industrial Technology Grant Program in 2011 from New Energy and Industrial Technology Development Organization (NEDO) of Japan.

\section{REFERENCES}

[1] M. J. Corley, and R. D. Lorenz, "Rotor Position and Velocity Estimation for a Salient-Pole Permanent Magnet Synchronous Machine at Standstill and High Speeds", IEEE Trans. Industry Applications, Vol. 34, No. 4, pp. $784-789$ (1998)

[2] Rahman, M.F., Zhong, L.,Khiang Wee Lim : "A direct torquecontrolled interior permanent magnet synchronous motor drive incorporating field weakening", IEEE Trans. Industry Applications, Vol.34, No.6, pp.1246-1253 (1998)

[3] M. Nasir Uddin, Tawfik S. Radwan, and M. Azizur Rahman, "Performance of interior permanent magnet motor drive over wide speed range", IEEE Trans. Energy Conversion, Vol.17, No.1, pp.79-84 (2002)

[4] Chan-Hee Choi, Jul-Ki Seok, and Lorenz, R.D. : "Wide-Speed Direct Torque and Flux Control for Interior PM Synchronous Motors Operating at Voltage and Current Limits", IEEE Trans. Industry Applications, Vol. 49, No.1, pp.119-117 (2013)

[5] P. M. Kelecy, R. D. Lorenz, "Control methodology for single inverter, parallel connected dual induction motor drives for electric vehicles," in Proc. IEEE PESC'94, pp.987-991 (1994)

[6] Matsuse, K. ; Kouno, Y. ; Kawai, H. ; Yokomizo, S.: "A speedsensorless vector control method of parallel-connected dual induction motor fed by a single inverter", IEEE Trans. Industry Applications., Vol.38, pp.1566-1571 (2002)

[7] J. D. Ma, B. Wu, N. R. Zargari, Steven C. Rizzo, "A Space Vector Modulated CSI-Based AC Drive for Multimotor Applications", IEEE Trans. POWER ELECTRONICS, VOL.16, NO.4, pp.535$544(2001)$

[8] A. Bouscayrol, M. Pietrzak-David, P. Delarue, R. Peña-Eguiluz, P. V. Kestelyn, "Weighted Control of Traction Drives With Parallel-Connected AC Machines", IEEE Trans. Industrial Electronics, Vol.53, No.6, pp.1799-1806 (2006)

[9] J, Itoh, N, Nomura, H, Ohsawa: "A Comparison between V/f Control and Position-Sensorless Vector Control for the Permanent Magnet Synchronous Motor", Proc. of the Power Conversion Conference PCC Osaka 2002, Vol. 3, pp.1310 - 1315 (2002)

[10] Nozawa, Y, et al : "Performance for Position Control of Two Permanent Magnet Synchronous Motors with the Five-Leg Inverter.", IECON'06, pp.1182-1187 (2006)

[11] Ibrahim, Z.; Lazi, J.M. ; Sulaiman, M.: "Independent speed sensorless control of dual parallel PMSM based on Five-Leg Inverter", Proc. of the 9th International Multi-Conference on Systems, Signals and Devices, pp.1-6 (2012)

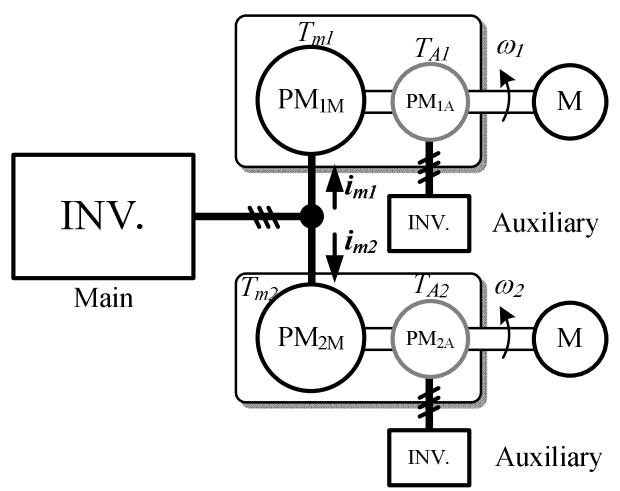

Fig. 9. Simulation model for parallel connected dual motor drive. In order to neglect the magnetic coupling, two parallel drive of the proposed system is validated using Fig. 8.

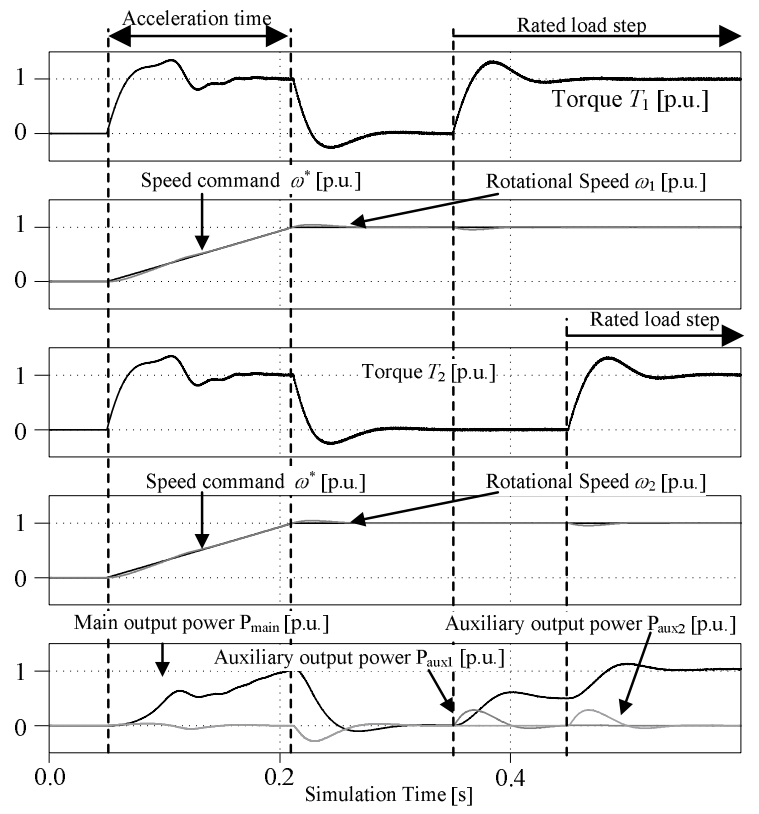

Fig. 10. Simulation results for parallel motor drive with damping control. Smooth acceleration progresses are confirmed in the two parallel connected PMSMs. After the rated motor speed, load step applies to each PMSM, stable operation of each PMSM can be confirmed.

[12] S. Mohammad et al.: "Space Vectors Modulation for Nine-Switch Converters", IEEE Trans. Power Electronics., Vol.25, pp.1488$1496(2010)$

[13] F. Gao, L. Zhang, D. Li, P. C. Loh, Y. Tang, H. Gao, "Optimal Pulsewidth Modulation of Nine-Switch Converter", IEEE Trans. Power Electronics, Vol. 25, No. 9, pp.2331-2342 (2010)

[14] Shibata, M., Hoshi, N.: "Novel inverter topologies for two-wheel drive electric vehicles with two permanent magnet synchronous motors", 12th European Conference on Power Electronics and Applications (2007)

[15] Mori, T., Tanaka, H., Kubo, Y., Matsuse, K., "Independent vector control of two permanent magnet synchronous motors fed by a four-leg inverter", Proc. of the 2012 IEEE International Conference on Power Electronics, Drives and Energy Systems (PEDES), pp.1-6 (2012)

[16] T. Nagano, et al. "Design of Multi-Parallel Drive Technique for System with Numbers of Permanent Magnet Synchronous Motors", The 10th IEEE International Conference on Power Electronics and Drive Systems, pp. 193-198 (2013)

[17] T. Nagano, et al. "Verification of Parallel Connected Multiple Motor Drive System with Numbers of Permanent Magnet Synchronous Motors", 15th European Conference on Power Electronics and Applications (2013) 